\title{
Place versus response learning in rats
}

\author{
Mark R. Cole, Amy Clipperton, and CARYN Walt \\ Huron University College, University of Western Ontario, London, Ontario, Canada
}

\begin{abstract}
In previous research designed to test whether place learning or response learning proceeds more quickly and better in rats, place has not been defined unambiguously when direction has been controlled by moving an apparatus around in the test room (Blodgett, McCutchan, \& Mathews, 1949; Skinner et al., 2003). In Experiment 1, we compared place and response learning while controlling direction in a static apparatus, thus making the meaning of place unambiguous. The performance of rats that had to make different turns to find food in a particular place and rats that had to always make the same turn to find food in two different places did not differ. In Experiment 2, visual cues were made equally discriminable for place and response learners in a static apparatus. Place learners still failed to outperform response learners, but there was evidence that response biases interfered more with place than with response learning. The results are discussed with reference to the historical debate that generated the original research and also in terms of more contemporary spatial-learning issues in rats.
\end{abstract}

Originally posed in the context of an examination of the veracity of two different learning theories - one presented by Hull (1943), which emphasized the learning of motor responses, and the other outlined by Tolman (1948), which emphasized the learning of environmental configurations, often called cognitive maps - the question of whether response learning or place learning is more primitive has proved difficult to answer clearly.

Tolman, Ritchie, and Kalish (1946) argued that they had provided a definitive answer to the question when they trained rats in a cross-maze located in a fixed place within a testing room and consisting of four arms radiating at $90^{\circ}$ angles from a cross-point, as shown in Figure 1A. The ends of the two shorter opposing arms were designated as start boxes, depicted as S1 and S2 in Figure 1A, whereas the ends of the other two opposing and longer arms were designated as goal boxes, shown as G1 and G2 in Figure 1A. Rats were assigned to be either place learners or response learners. All the rats started from S1 or S2 on different randomly selected trials with either $\mathrm{G} 1$ or $\mathrm{G} 2$ baited with food. Place learners always found the food in one place (e.g., in G1) and had to make either left or right turns to get there, depending on the start point. Place learners were always required to go to their nonpreferred place, as determined by a preliminary test trial. Response learners had to make the same response (always a right turn) and, thus, found food in G1 when starting from S1 and in G2 when starting from S2. Oddly, in view of the use of a nonpreferred place for place learners, there was no similar attempt to control response preferences for response learners.

Tolman et al. (1946) reported that all 8 place learners quickly reached a criterion of 10 correct trials in a row, whereas 5 of 8 response learners failed to reach the same criterion within 72 trials, and the 3 that did reach the cri- terion all took more trials than the slowest place learner. Tolman et al. interpreted these results as direct support for the hypothesis that learning to go to a particular place is easier than learning specific motor responses, such as turning right, and as indirect support for his cognitiveleaning theory.

Blodgett, McCutchan, and Mathews (1949) noted that Tolman et al. (1946) may have inadvertently given place learners an unfair advantage because, in always going to one place - for example, to G2 in Figure 1A-they were also always going in a constant compass direction. By contrast, response learners were required to go in opposite compass directions on different trials. According to Blodgett et al., this confound between direction and place actually made it impossible to determine whether the results were due to a place-learning predisposition, a direction-learning predisposition, or both.

Blodgett et al. (1949) exposed hungry rats to a T-maze and, by systematically varying the location and, sometimes, the orientation of the maze within the room and also varying which goalbox contained the food reward, were able to examine pure place learning, pure response learning, and pure direction learning. Figure 1B shows the four placements and orientations for the maze. One of the place/orientations is depicted using a solid line to emphasize the fact that this research employed a T-maze in four possible place/orientations, not a cross-maze in two possible places. Pure place-learning rats were started on different trials from $\mathrm{S} 1$ or $\mathrm{S} 3$ and always found food at G2. In going to $\mathrm{G} 2$ from $\mathrm{S} 1$ or $\mathrm{S} 3$, the rats in this group made different responses (left and right turns) and went in different compass directions. It is important to note, however, that the approach taken by Blodgett et al. defined place as a location in the larger environment (i.e.,

M. R. Cole, mcole@uwo.ca 
A

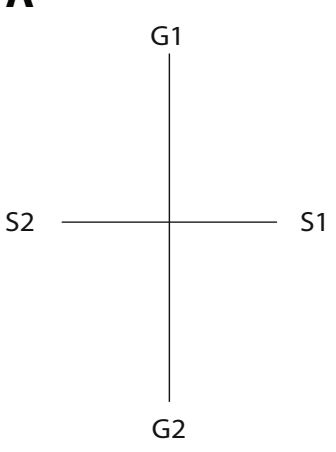

C

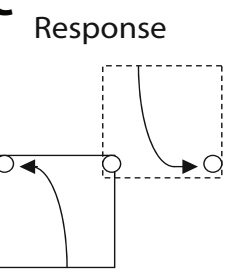

B

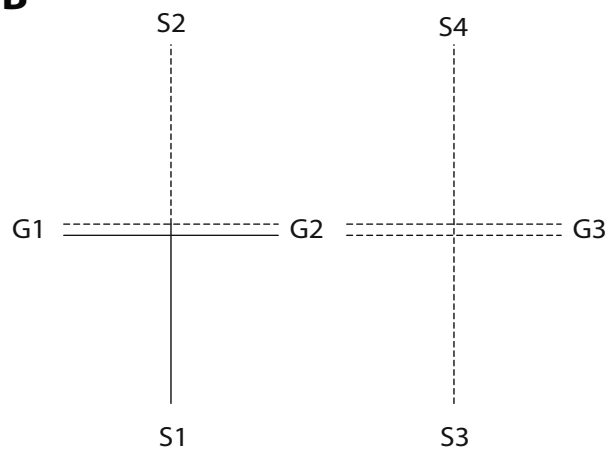

Place

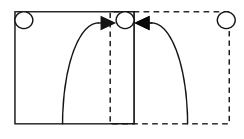

Direction

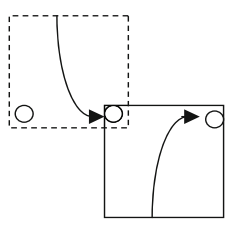

D

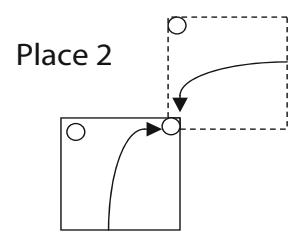

Figure 1. (A) Cross-maze used by Tolman, Ritchie, and Kalish (1946), showing opposite goals G1 and G2 and opposite start points S1 and S2. (B) Locations and orientations of the T-maze used by Blodgett, McCutchan, and Mathews (1949), with G1 through G3 representing goal locations in the room and S1 through S4 representing starting points. (C) Locations and orientations of the platform used by Skinner et al. (2003) in Experiment 1. (D) Locations and orientations of the platform used by Skinner et al. in Experiment 2.

$x$ meters from the north wall and $y$ meters from the east wall), not as a place in the T-maze (e.g., the end of the right arm). Pure response learners had to make a constant turning response that was opposed to their choice on the first trial. In order to find food, five of the pure response learners had to make left turns in going from S1 to G1 or from $\mathrm{S} 2$ to $\mathrm{G} 2$ on different trials, whereas the other 3 had to make right turns in going from $\mathrm{S} 1$ to $\mathrm{G} 2$ or from $\mathrm{S} 2$ to G1 on different trials. Again, regardless of which type of turn was required, to be rewarded, the rats had to go to different places, at least with respect to the room, and in different compass directions on the two types of trials. Pure direction learners had to go in the direction opposite to that chosen on the first trial. To find food on these two randomly presented types of trials, 5 rats had to move in a constant compass direction after the choice point in going from S1 to G1 or from S4 to G2, thus making left and right turns, respectively, and going to different places with respect to the room. The other 3 pure direction learners were required to go in the opposite compass direction after the choice point, either from S1 to G2 or from S4 to G3, and thus make right and left turns and go to different places in the room to find food on these two types of trials.
After 48 trials at the rate of 4 trials per day, pure place learners had made significantly more incorrect choices than had either pure response learners or pure direction learners, which themselves did not differ significantly. Blodgett et al. (1949) concluded that direction learning had been mainly responsible for the good performance by the place learners in the experiment by Tolman et al. (1946) and that response learning was more easily acquired than place learning when direction was partialed out.

In the first of several experiments, Skinner et al. (2003) carried out what was, in essence, a replication of the research by Blodgett et al. (1949). They tested hungry rats, using what they called an open-field maze instead of a T-maze. Although Blodgett et al. had used an elevated maze without any sidewalls, Skinner et al. argued that a $1.0 \times 1.0 \mathrm{~m}$ elevated platform would give their rats an even better view of their environment and would thus provide a setting conducive to the formation of a cognitive map. They bolstered their argument by noting that research examining hippocampal cell activity had shown evidence that, whereas open-field settings led to the formation of a single cognitive map, more restricted pieces of apparatus such as the T-maze tended to produce multiple maps 
(Markus et al., 1995; McNaughton, Barnes, \& O'Keefe, 1983; Muller, Kubie, \& Ranck, 1987). Like Blodgett et al., Skinner et al. moved the platform, which had food cups in two adjacent corners, about in the room on different trials to enable a separate analysis of pure place, pure response, and pure direction learning. Figure $1 \mathrm{C}$ shows examples of how the apparatus was positioned and oriented on different trials for each type of learning, with one position/ orientation represented by the solid outline and the other by the dotted outline. The arrows show the starting points and path apparently taken to arrive at the baited food cup.

Response learners reached a criterion of $18 / 20$ correct choices significantly more quickly than did direction learners, which in turn reached criterion more quickly than did place learners. In fact, not a single place learner reached the criterion in 300 trials! With respect to the comparison between place learners and response learners, these results replicated those in Blodgett et al. (1949).

\section{EXPERIMENT 1}

As was mentioned in the general introduction, in the research by both Blodgett et al. (1949) and Skinner et al. (2003), direction was controlled by changing the position and orientation of the apparatus within the room on different trials. In the process, both of these research teams defined place in terms of its coordinates within the room, rather than with respect to the apparatus. Gallistel (1990) has argued that confusion concerning the meaning of place in research such as that conducted by Blodgett et al. and Skinner et al. has not helped in the attempts to provide a clear answer to the question of whether response learning or place learning is more easily acquired. For Tolman et al. (1946), this was not an issue, because place with respect to the room and place with respect to the apparatus were the same, given that the apparatus remained static. If the rats in the experiments reported by Blodgett et al. and Skinner et al. had used only extramaze cues in solving the place problem, there would have been no difficulty for them. Defined as a location in the room, place would have been unambiguous and fixed. However, had their place learners used intramaze cues instead of, or in combination with, extramaze cues, place would not have been constant at all; rather, the rats would have found food at the end of the right arm (Blodgett et al.) or in the right corner (Skinner et al.) in one placement and at the end of the left arm (in the left corner) in the other placement. Thus, had place learners in these experiments relied on intramaze cues to solve the place problem, they would have found it very difficult. There is evidence that rats make use of both types of cues in spatial learning and that it is difficult to predict exactly what cues they will make use of in a given situation (Cheng \& Spetch, 1998; Gallistel, 1990; Save, Poucet, \& Thinus-Blanc, 1998). It should also be noted that, to the extent that they did use intramaze cues, the response learners might have had an advantage in the experiments by both Skinner et al. and Blodgett et al. Whereas the response learners found food in different places in the room on each type of trial, it was in the same place (e.g., at the end of the left arm or in the right corner) within the apparatus. Thus, it is possible that these rats had the advantage of both making a constant response and going to a constant place.

The purpose of the first experiment in the present research was to replicate the experiment carried out by Blodgett et al. (1949) and the first experiment carried out by Skinner et al. (2003), but without the potential confusion regarding the meaning of place that occurs when an apparatus is moved about within a larger experimental space in order to control direction. This was accomplished by fixing place with respect to both the local apparatus and the larger experimental environment.

\section{Method}

\section{Subjects}

Fifteen male hooded rats of the Long-Evans strain, obtained from Charles River in Montreal, were used. The rats had all had prior experience in operant chambers, on a small eight-arm radial maze, and in a pilot study that was conducted just before the present experiment. The rats were reduced to $85 \%$ of free-feeding body weight and were housed individually in 2-rat shoe-box style cages with ad lib water. The cage room was maintained at $23^{\circ} \mathrm{C}$ with a $12: 12$-h light-on:lightoff cycle. The lights were red during the first and last hours of the lighted period. Testing was carried out during the light-off phase.

\section{Apparatus}

As is shown in Figure 2, the apparatus consisted of four food towers set $90 \mathrm{~cm}$ apart on center and arranged along the long axis of an oval arena bounded by a wall constructed from Masonite that was $40 \mathrm{~cm}$ high. The long axis of the maze ran north-south. From left to right, as is shown in Figure 2, the towers were designated A, B, C, and $\mathrm{D}$, respectively. Each food tower consisted of a block of cedar $10 \times 10 \times 20 \mathrm{~cm}$ high and coated with Varathane. A plastic food cup, $2.5 \mathrm{~cm}$ in diameter, $1.9 \mathrm{~cm}$ deep, and created from a $35-\mathrm{mm}$ film canister, was attached by means of a small screw to the center of the top of each tower. Due to the height of the towers, a rat could not investigate the contents of a food cup without rearing up on the tower on which it was mounted, an action that was unmistakable for datarecording purposes. Three disks, each $2.6 \mathrm{~mm}$ in diameter and each containing ten 3-mm-diameter holes, were fashioned from the lids of $35-\mathrm{mm}$ film canisters. Pressing one of these tightly fitting disks down into the food cup made it possible to place inaccessible bait in the food cup on top of any tower. Thus, to control for odor cues, all four towers always contained bait, but the bait was accessible only on the "correct" tower on a given trial. The maze was located in the center of a windowless room measuring $4.5 \times 5.0 \times 2.5 \mathrm{~m}$ high. The northeast corner of the room had an alcove beyond this perimeter that measured $1.5 \times 0.6 \mathrm{~m}$. The walls of the room were painted yellow, and there were three, two, and three gray-painted doors set into the east, south, and north walls, respectively, whereas the center of the west wall was the location of a large stainless steel sink. The rightmost door on the east wall was always open, whereas the rest of the doors were always closed. A white lab coat hung on the inside of the open door, thus making the southeast corner of the room visually distinctive. There was a $1.0-\mathrm{m}$-high white freezer located in the southwest corner of the room, and there was also a brown cube refrigerator on top of a table, situated in the northwest corner of the room. Cubes of Black Diamond Medium Cheddar Cheese measuring $1 \mathrm{~cm}^{3}$ were used to bait the towers. The room was well illuminated by four light fixtures mounted on the ceiling, each of which contained four $120-\mathrm{cm}$-long fluorescent tubes behind a translucent plastic lens.

\section{Procedure}

Preliminary training. The rats were initially trained to eat from the food towers by placing three baited training towers that were 10 , 15 , and $20 \mathrm{~cm}$ high, respectively, along the long axis of the arena. 
A

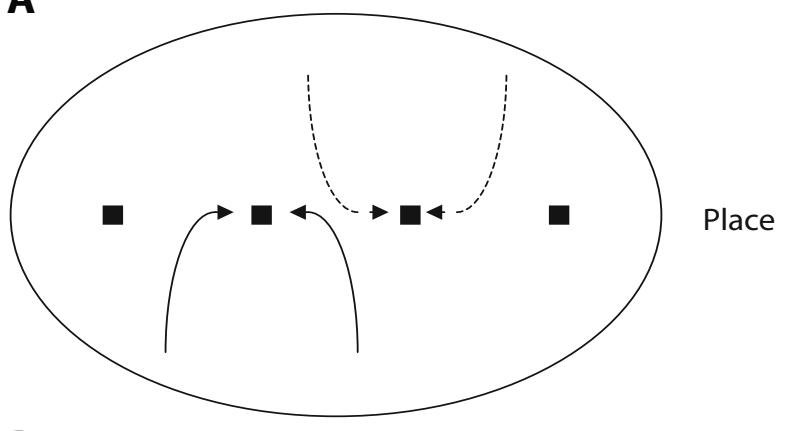

B

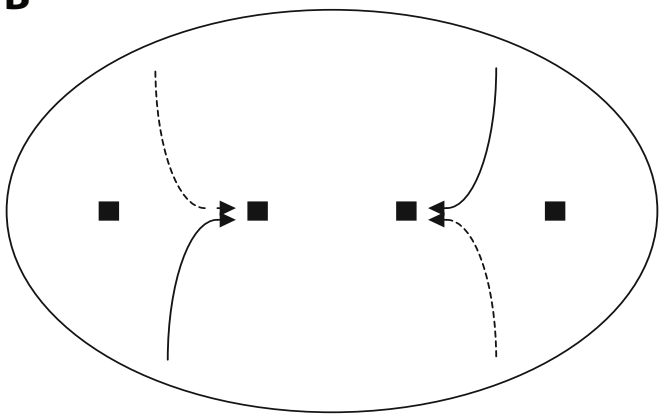

C

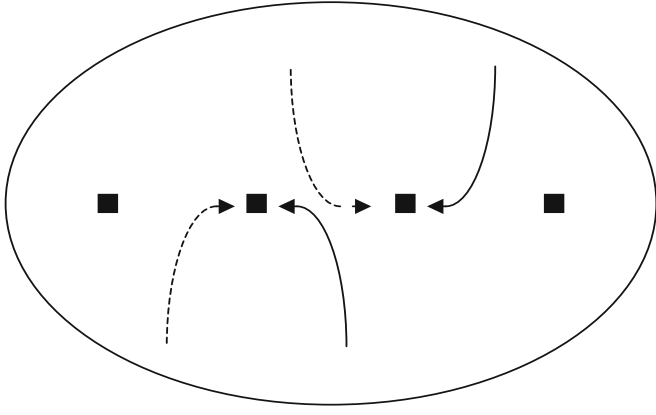

Figure 2. Arrangement of food towers within the oval arena used in Experiment 1, also showing the starting points and paths taken to get to the baited tower on the two types of trials for place (A) response (B), and direction (C) learners. The location of the baited tower is indicated by the solid arrows for some rats in each group and by the dotted arrows for the rest of the rats in each group.

The rats were then placed one at a time into the arena and left there until they had visited all three towers. After five such trials, all the rats went to all three towers, reared up, and removed and ate the cheese contained therein.

Training. Following preliminary training, the rats were randomly assigned to one of three groups $(n=5)$ and were given four trials per day, two in the morning and two in the afternoon, with the apparatus set up as shown in Figure 2. Successive morning or afternoon trials were separated by at least $1 \mathrm{~h}$. On all trials, the food cups on the outer two towers were charged with inaccessible cheese and were never correct. On any given trial, although one of the two inner towers also contained inaccessible cheese, the other one had accessible cheese (henceforth referred to as the baited tower). A 20-item sequence of start points and corresponding baited towers was selected randomly by a computer program prior to the start of training. Within the 20-item sequence, each of the two alternatives was represented 10 times. The rat remained in the apparatus until it found the baited tower or until $3 \mathrm{~min}$ had passed. On each trial, the rat started from one of the two randomly selected starting points with one of the inner pair of towers baited as shown in Figure 2. Three of the rats in each group could find the baited tower by following the paths indicated by the solid arrows, whereas the other 2 rats in each group were required to find food on the tower indicated by the dotted arrows. Thus, as is shown by the solid arrows in Figure 2A, 2 place rats always went to Tower $\mathrm{B}$ to find food, turning in different directions (right vs. left) and going in different directions (north vs. south) from the two different starting points. The dotted curves in Figure 2A show that the other 3 place rats always found food on Tower $\mathrm{C}$, again by making different turns and going in different directions on the two types of trials. As is shown by the solid arrows in Figure 2B, 3 response rats always had to turn right, thus going in different directions (north or south) to arrive at different towers (B or C) from the two different starting points to find food. The other 2 response rats could follow the path traced by the dotted arrows in Figure 2B, turning to the left but going in different directions to find food located on different towers on the two types of trials. Finally, as is depicted by the solid arrows in Figure 2C, 3 direction rats always had to bear south to arrive at different baited towers (B or C) while making different turns (left or right) to find food on the two types of trials, whereas the other 2 direction rats could follow the paths indicated by the dotted arrows, bearing north, but again making different turns to get to different baited towers. Whereas the open-field design of the apparatus did not force the rats to actually follow the paths indicated in Figure 2, observation revealed that they did so. Also, it should be noted that from the indicated starting positions, all of the rats had a very good view of the larger testing environment. All of them would have been able to see at least the upper half of all the walls and, depending on which way they were facing, such features as the sink, the cube refrigerator, the lab coat, and the top portion of the freezer. After being removed from the maze in the afternoon session, the rats were weighed and given a ration of lab chow sufficient to maintain their body weights at $85 \%$ of their original free-feeding weights.

All the rats were trained for 150 trials and then terminated if they had by then met a performance criterion of $18 / 20$ correct choices. The rats that had not yet met the criterion within 150 trials were given a further 30 trials.

Data recording. On each trial, after placing the rat in the arena facing the line of towers, the researcher stood outside the arena near the release point to record the data, using a lectern. Facsimiles of the maze were used to record the order of tower visits.

\section{Results}

One of the direction rats became ill and had to be destroyed during the experiment, and so none of the data from that rat are included in the analyses.

\section{Percentages of Correct Choices}

Percentages of correct first choices by the rats over the first 15 blocks of 10 trials are shown in Figure 3. A $3 \times$ 15 ANOVA revealed a significant main effect of blocks of trials $[F(14,154)=16.7, p<.05]$, whereas the group effect approached, but did not quite achieve, significance $[F(2,11)=3.3, p=.08]$.

\section{Trials to Criterion}

One place learner and 2 direction learners failed to reach the criterion of $18 / 20$ correct responses within 150 trials and were given an additional 30 trials but still failed to reach the criterion. Accordingly, further training of these 3 rats was terminated, and each was assigned a trials-to-criterion score of 198, the score that they would have achieved had they been correct on Trials 181-198. Response learners had the fewest trials to criterion $(M=66.4, S D=37.0)$, followed by place learners $(M=109.6, S D=51.0)$, followed by direc- 


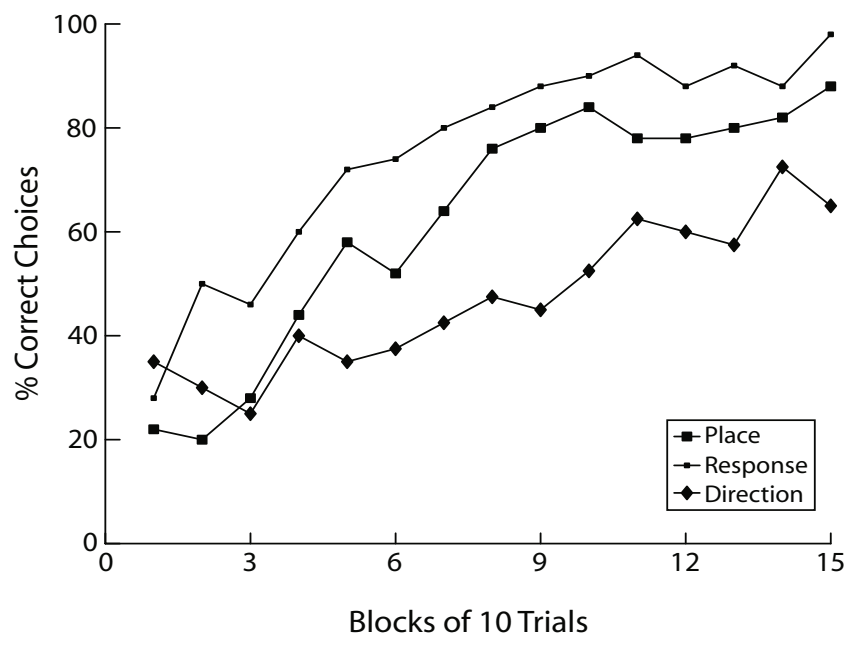

Figure 3. Percentages of correct first choices in blocks of 10 trials for place, response, and direction learners in Experiment 1.

tion learners $(M=165.8, S D=42.3)$. A one-way ANOVA revealed a significant effect of groups $[F(2,11)=8.4, p<$ $.05]$. Planned comparisons revealed that whereas both place rats $[t(7)=2.6, p<.05]$ and response rats $[t(7)=3.6, p<$ $.05]$ outperformed the direction rats, there was no significant difference between the place rats and the response rats.

\section{Discussion}

The results of Experiment 1 suggest that there may have been confusion about the meaning of place in the previous research designed to control direction by utilizing a movable apparatus. Both Blodgett et al. (1949) and Skinner et al. (2003), in their first experiments, found poor performance by place learners, relative to response learners. In fact, Skinner et al. reported that not even 1 of their place learners reached the criterion of 18 out of 20 correct choices within 300 trials. Experiment 1 in the present research showed that all but 1 of the place learners reached this criterion within 150 trials and performed at least no worse than response learners when place was constant with respect to both the local apparatus and the larger experimental environment. Moreover, the present data are in agreement with those in Skinner et al. in showing very poor performance by pure direction learners. This is in sharp contrast to the data reported by Blodgett et al., who found direction learners to be best among the pure learners.

\section{EXPERIMENT 2}

Although not commenting on the ambiguity concerning the definition of place in the movable apparatus approach to controlling direction, Skinner et al. (2003) did point out another possible way in which this tactic may have left pure place learners at a disadvantage. They noted that whereas both response and direction learners started on opposite sides of the room on their two types of trials, place learners always started from the same side and, moreover, the start points were very close together. Thus, they suggested that place learners might have been af- forded less discriminable views of the extramaze environment on the two types of trial than were either response or direction learners. More specifically, they argued that the low discriminability of the start points for place learners might have interfered with their ability to create cognitive maps and, hence, their ability to solve the place problem.

To test this hypothesis, in a second experiment, Skinner et al. (2003) compared pure direction learners with two groups of pure place learners. One group of place learners (Place 1) were treated exactly like the place learners in the first experiment, in that they started from closely separated points on the same side of the room. A second group of place learners (Place 2), however, started from points $90^{\circ}$ apart, as shown in Figure 1D. Although likely not as discriminable as the starting points of the direction learners, which were $180^{\circ}$ apart, Skinner et al. argued that the starting points for Place 2 rats should have been more discriminable than those for Place 1 rats. Results from this second experiment showed that Place 1 learners performed as poorly as had the place learners in Experiment 1 and much worse than the Place 2 learners, which did as well as the direction learners. It is unfortunate that a group of response learners was not tested in this experiment, but in a third experiment reported by Skinner et al., Place 1 learners performed more poorly than either Place 2 learners or response learners, which themselves did not differ significantly. Of course, it may be assumed that the place learners in Blodgett et al.'s (1949) research were also likely put at a disadvantage by having had very similar starting points and that this might have contributed to their poor performance, relative to response learners.

In Experiment 2 of the present research, we sought to control discriminability of starting points, while continuing to define place as a fixed location within both the immediate apparatus and the larger experimental space. The hypothesis, based on the ideas advanced by Tolman (1948), was that, without confusion as to the meaning of place and with starting points as discriminable as those for response learners, place learners should actually outperform response learners, even when direction was controlled. Due to the poor performance by the direction learners in Experiment 1, and to improve the power of the statistical analyses, only pure response and pure place learners were tested in Experiment 2.

\section{Method}

\section{Subjects}

Fifteen male hooded rats of the Long-Evans strain, obtained from Charles River in Montreal, and with prior experience both in operant chambers and on a small eight-arm radial maze, were used. The rats, which were not the same rats as those used in Experiment 1, were reduced to $85 \%$ of free-feeding body weight and were housed as were the rats in Experiment 1: The rats were again tested during the light-off portion of the 12:12-h light-on:light-off cycle.

\section{Apparatus}

In Experiment 2, the same four food towers were used, but instead of placing them along a straight line, they were arranged in a square pattern with $120 \mathrm{~cm}$ between the centers of adjacent towers. The four towers were surrounded by a Masonite wall that was $40 \mathrm{~cm}$ high. The wall enclosed a circular area $3.0 \mathrm{~m}$ in diameter, as shown 
in Figure 4A. The apparatus was set up in the same room, with the same features and under the same general conditions as those that had been in effect for Experiment 1. Beginning with the tower in the southeast corner, shown in Figure 4A, and moving in a clockwise fashion, the towers were designated A, B, C, and D, respectively, with the starting points numbered 1 through 4 , also in a clockwise fashion beginning at the east side of the circle. As was the case in the first experiment, all of the rats had an unimpeded view of at least the upper half of the walls of the test room from their starting points. Also, as in the first experiment, cheese cubes were used to bait the towers.

\section{Procedure}

Preliminary training. The rats were initially trained to eat from the food towers by placing three baited towers that were 10,15 , and $20 \mathrm{~cm}$ high, respectively, haphazardly within the circular arena. The rats were then placed one at a time into the arena and left there until they had visited all three towers. After five such trials, all the rats reared up, removed, and ate the cheese contained on all three towers.

Training. Following preliminary training, the rats were randomly assigned to be place learners or response learners $(n=8$ and $n=7$, respectively) and were given four trials per day, two in the morning and two in the afternoon. As in Experiment 1, successive morning or afternoon trials were separated by at least $1 \mathrm{~h}$. On any trial, the food cups on three of the four towers were charged with inaccessible cheese and represented incorrect choices, whereas one tower contained accessible cheese (the correct tower). Each rat remained in the apparatus until it found the baited tower or until $3 \mathrm{~min}$ had passed. On each trial, the rats started from one of two randomly selected starting points, with one of the pair of towers adjacent to that starting point baited, as shown in Figures 4B and 4C. Thus, to find food, 4 of the response rats always had to make a right turn when leaving Starting Point 1 or 4 on different trials, going to different towers (D or C) and in different directions (north or west), as indicated by the solid arrows in Figure 4B. The 3 remaining response rats always had to turn left to find food on different towers while going in different directions on the two types of trials, as indicated by the dotted arrows in Figure 4B. Similarly, 4 of the place rats departed from Starting Point 1 or 4 on different trials to find the baited tower (always D), making different turns (right or left) and going in different directions (north or east), as indicated by the solid arrows in Figure 4C. The other 4 place rats were required to find food on the baited tower, as indicated by the dotted arrows in Figure $4 \mathrm{C}$, going in different directions and making different turns from the two starting points. Once again, in all cases, the curved arrows represent the actual paths taken by the rats, based on observation. A 20 -item sequence of start points and corresponding baited towers were selected randomly by a computer program prior to the start of training for each squad in each of the two groups. After being removed from the maze following the afternoon session, the rats were weighed and given a ration of lab chow sufficient to maintain their body weight at $85 \%$ of the original free-feeding weight.

All the rats were give 150 trials and then terminated if they had met a performance criterion of 18/20 correct choices. Any rat that had not yet met the criterion within 150 trials was given 30 additional training trials.

\section{Results \\ Percentages of Correct Choices}

Figure 5 shows percentages of correct first choices over 15 blocks of 10 trials each. Although it appears from the graph that the place learners enjoyed a slight advantage over the response learners, particularly toward the end of training, a $2 \times 15$ ANOVA revealed only a significant effect of trial block $[F(14,182)=14.5, p<.05]$. A separate $2 \times 5$ ANOVA conducted on the last five blocks of 10 trials re-
A

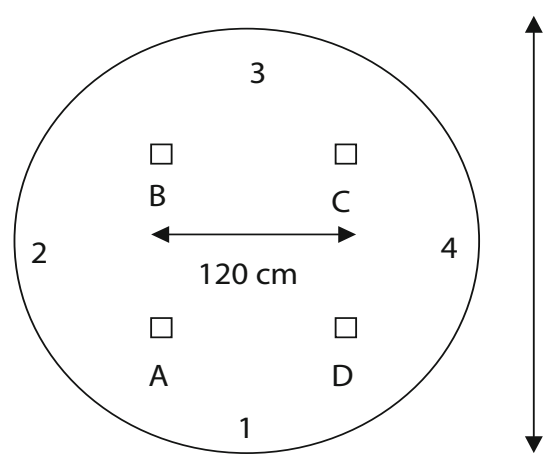

$3.0 \mathrm{~m}$

B

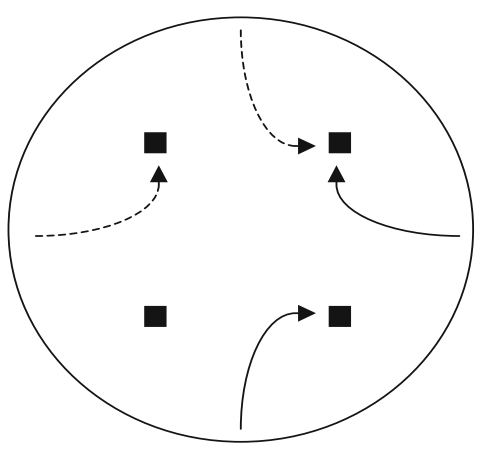

Response

C

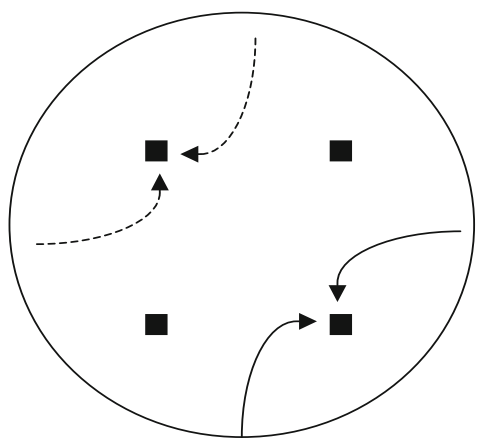

Place

Figure 4. Arrangement of the food towers in Experiment 2 (A), and the starting points and paths taken to get to the baited tower on the two types of trials for response learners (B) and place learners $(\mathrm{C})$. The location of the baited tower is indicated by the solid arrows for some rats in each group and by the dotted arrows for the rest of the rats in each group.

vealed an effect of group that approached but did not quite achieve statistical significance $[F(1,13)=3.04, p=.10]$.

\section{Trials to Criterion}

One response rat failed to reach the criterion of 18/20 correct responses within 150 trials and was given an additional 30 trials without showing any improvement. Accordingly, this rat was assigned a trials-to-criterion score of 198. There was little difference between the trials-tocriterion scores of the place learners $(M=77.9, S D=20.8)$ and response learners $(M=79.3, S D=67.7)$, an observation confirmed by the results of a one-way ANOVA, which failed to show a significant effect of groups $[F(1,13)=$ $0.003, p>.05]$. One interesting aspect of the trials-tocriterion data, as evidenced by the standard deviations, 


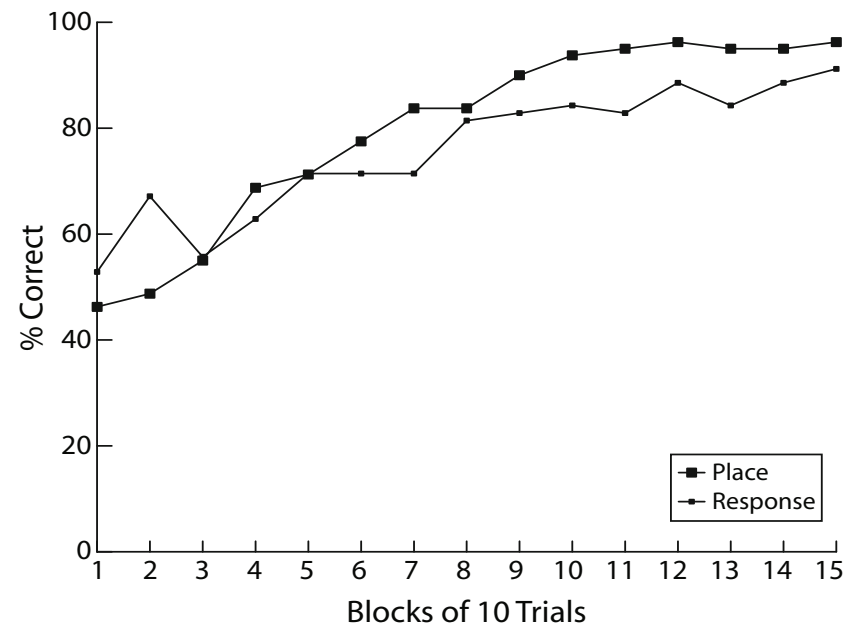

Figure 5. Percentage of correct first choices in blocks of 10 trials for place and response learners in Experiment 2.

is that the results for the response rats were much more variable than those for the place rats. The response rats numbered among them not only the single slowest learner, with an estimated score of 198 trials to criterion, but also the 2 fastest learners, with trials-to-criterion scores of 20 and 29 , respectively.

\section{Error Analysis}

An analysis was undertaken of the errors committed by the 3 rats in each group, the performance of which was worse than the median for their respective group. Separately, from each of the two starting points, the first tower selected on incorrect trials was noted over all such trials. These data are shown in Figure 6. Two alternative hypotheses were tested: (1) that the rats had a place preference that interfered with the required choice; or (2) that the rats had a response preference that interfered with the required choice. A place bias would be indicated by the selection of the same tower on all error trials, regardless of starting point or group assignment. None of the 6 rats, whose data are depicted in Figure 6, showed this pattern.

A response bias would be shown in place learners by the selection, on error trials, of a single tower from one of the two starting points, but not from the other. All 3 of the weak place learners showed such a pattern of incorrect choices. For example, Rat 4 usually went left from Start 3 to Tower C. Note that a similar left turn from Start 2 led to Tower B, which was the correct tower for this place learner. As a result, there were few errors of any sort for Rat 4 from Start 2. Rats 5 and 13 also demonstrated a tendency to make left turns, but from Start 1 and Start 4, respectively. Thus, a left turn from Start 4 brought them to Tower $\mathrm{D}$, their correct place destination, but led them to Tower A, an incorrect choice, when they departed from Start 1. Goodness-of-fit tests revealed that the distributions of errors from Start 3 (Rat 4) and from Start 1 (Rats 5 and 13) were unlikely to have been due to chance $\left(\chi^{2}=36.8,16.9\right.$, and 27.9, respectively).

The error distribution predicted by a response bias for response learners is different. A response learner with a tendency to make the required response should make few errors, but one with a bias toward the response not demanded should make many errors, but to different towers from the two start points. Two of the 3 response learners that performed worse than the median showed such a pattern. Rat 6 went most often to Tower A from Start 2 and to Tower B from Start 3, in both cases making a right turn, whereas a left turn was the correct response for this rat. Rat 12 , for which the correct response was a right turn, tended to make incorrect left turns, going to Tower A from Start 1 and Tower D from Start 4. The distributions of incorrect responses for Rat 6 , leaving from both Starts 2 and 3, respectively, differed significantly from chance, using a goodness-of-fit analysis $\left[\chi^{2}(2)=22.7\right.$ and 16.6, $\left.p<.05\right]$. Similarly, the distribution of incorrect responses for Rat 12, leaving from Starts 1 and 4, respectively, were also unlikely due to chance $\left[\chi^{2}(2)=76.2\right.$ and 14.0, $\left.p<.05\right]$. Although Rat 17 showed a right turn bias from Start $3\left[\chi^{2}(2)=31.1, p<.05\right]$, it failed to show a similar tendency to go right to Tower A from Start 2.

\section{Discussion}

Even under conditions that should not have "stacked the deck" either for or against place learners, there is little support for the hypothesis originally advanced by Tolman et al. (1946) that place learning is easier than response learning for rats. The finding that the percentage of correct choice data did not show a significant effect of group even during the last 50 trials was further compromised by the failure to see any difference at all between place and response learners when trials to criterion are considered, although such an all-or-none measure is arguably less sensitive than percentage of correct choices.

The error analysis lends support to the conclusion that rats bring response biases to this learning situation. These biases would have affected response learners in different ways. On the one hand, they were likely responsible for the very rapid attainment of the criterion of 18/20 correct responses for the rats required to make the very response toward which they were biased. Being correct on most, if not all, trials from the outset should have resulted in repetition of that response, due both to the ongoing bias and to the continuous reinforcement provided by the choice made as a result of the bias. On the other hand, the same biases likely produced much slower attainment or, in one case, no attainment at all of criterion, when the response demanded was opposite to that favored by a rat. Being incorrect on most, if not all, trials from the outset might be expected at some point to lead to at least occasional switches to the nonpreferred response and, as a result, reinforcement of those responses. But if the bias were strong, and the error analysis data suggested that it was for 2 of the 3 response rats that made a lot of errors, the nonpredisposed response might not be expressed often enough to become dominant, even though reinforced when it did occur. However, with only 2 of the 3 rats that made a lot of errors showing clear evidence of a contrary bias, it might be assumed that there were more instances of response bias favorable 


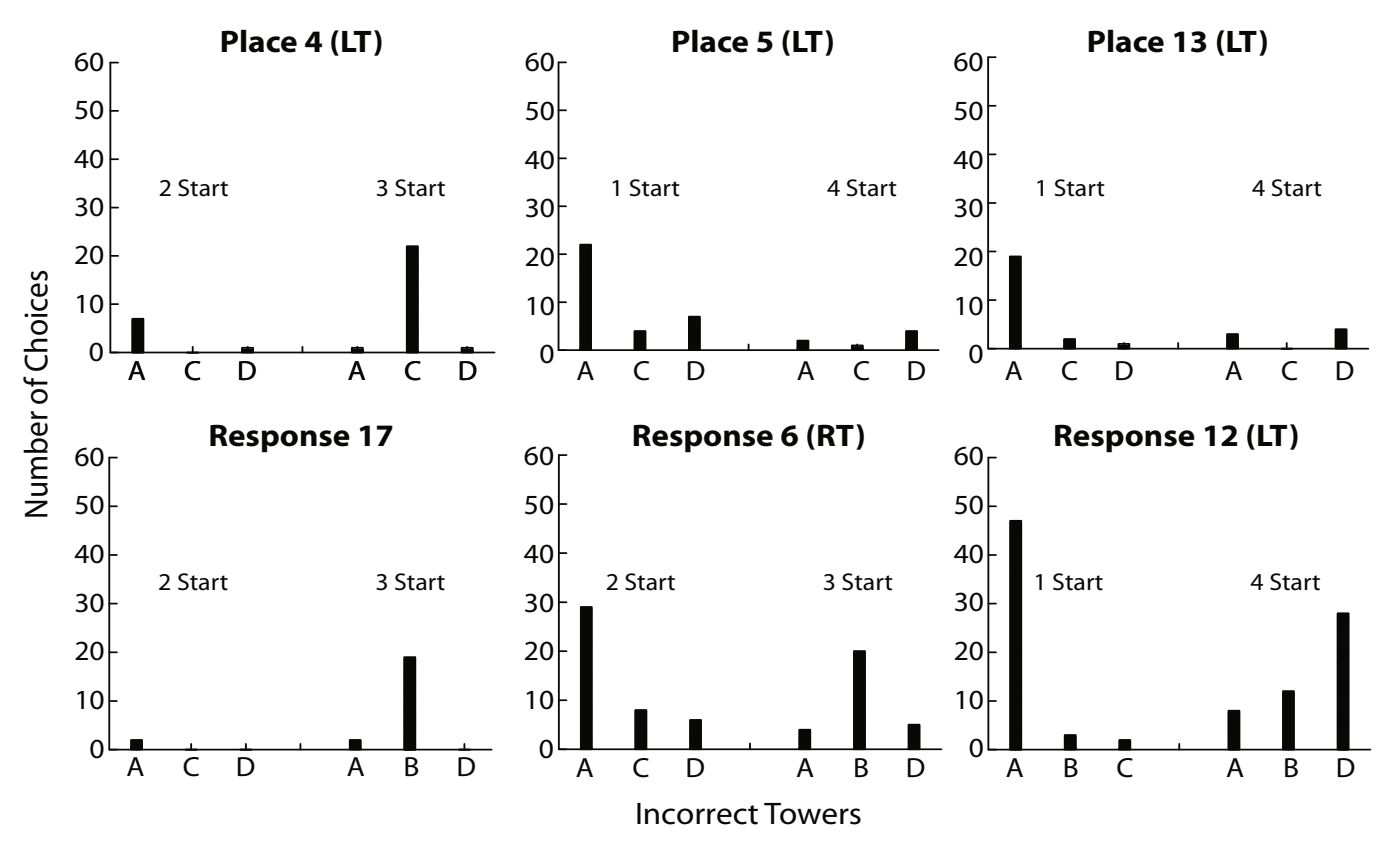

Figure 6. Frequency distribution of incorrect first selections by place and response learners performing below the median of trials to criterion in Experiment 2.

to acquisition of the required response and, hence, that the response learners as a group may have been assisted by, more than hindered by, response biases. In the case of place learners, the implications of a response bias are different. Regardless of whether a place learner had a right or a left bias, making such a response on every trial would lead to $50 \%$ success. Moreover, being successful on half the trials might well tend to lead to perpetuation of the biased response. Thus, unlike in the case of response learners, for which a response bias would have been helpful or detrimental, depending on whether the required response was the one the rat was inclined toward, response biases likely impeded learning for all the place learners. Wike, Kintsch, and Remple (1959) showed that rats reinforced $50 \%$ of the time for correct turns in a T-maze showed much slower acquisition of correct responding than did rats reinforced for every correct response and, moreover, the deficit was still present after 48 trials. So, assuming at least some response bias among the place learners and, furthermore, given that such biases should always impede place learning to some extent, it could be assumed that response biases negatively affected place learners as a group more than response learners as a group. This alone might explain why, even under ideal circumstances for the formation of cognitive maps, place learners still did not outperform response learners.

\section{GENERAL DISCUSSION}

In an attempt to ensure that compass direction was not confounded with place or response learning, as had been the case in an earlier experiment conducted by Tolman et al. (1946), Blodgett et al. (1949) and, later, Skinner et al. (2003) used a movable apparatus that left place ambiguous. As a result, in these studies, place was fixed only with respect to the larger experimental environment, not with respect to the apparatus that moved about within it. Experiment 1 in the present research was designed to extend the work by Blodgett et al. and Skinner et al., controlling direction, but using a fixed apparatus so that the meaning of place remained unambiguous. That is, place within the local apparatus and place within the larger experimental space were the same. Both Blodgett et al. and Skinner et al., in their first experiments, had shown that place learners performed significantly worse than response learners. However, Experiment 1 in the present research revealed that, with compass direction controlled and the meaning of place unambiguous, place learners performed at least no worse than response learners. This outcome is consistent with the hypothesis that there was confusion about the meaning of place in the experiments reported by Blodgett et al. and Skinner et al. That said, the first experiment in the present research failed to show that place learning was actually superior to response learning, and as a result, we failed to replicate the findings of Tolman et al. when the confound between place and compass direction had been eliminated.

In retrospect, the issue of compass direction that stimulated much of the research cited may not have been as serious a difficulty as first suggested by the results of Blodgett et al. (1949). To date, theirs is the only research addressing the question of response versus place learning that has shown that direction learners perform well. Both Skinner et al. (2003) and the first experiment in the present research showed that direction learners perform poorly, relative to response and/or place learners. According to Blodgett et al., three of the four walls in their laboratory had large windows that supplied natural light and 
that may have provided very salient direction cues. From their description, Skinner et al. had a laboratory space quite similar to ours, without windows, having uniformly painted walls with few decorations, and illuminated by fluorescent lighting. It is ironic that the effort to control a variable that may not be much of a factor in most modern animal laboratories may have led to a new and worse confound concerning the meaning of place that has since compromised comparisons between response learning and place learning.

It is likely that the place rats in Experiment 1 of the present research were also hampered by having to depart from start points that provided less discriminable views of the larger environment. Skinner et al. (2003) showed that when the view of the environment provided from the start points for place rats was made as discriminable as that for the response rats, place rats did much better than they had done when this was not the case, although they still did not perform better than response rats. We hypothesized that the failure of their place rats to outperform their response rats may have been due to the confusion concerning the meaning of place in that research. Experiment 2 in the present research was designed to make the start points and, hence, the views of the environment of the place learners the same as those of the response learners, while continuing to avoid the use of a movable apparatus as a means of controlling direction and, hence, eliminating the possibility of confusion about the meaning of place. However, even under these seemingly ideal conditions, place learners still failed to outperform response learners.

Perhaps the most interesting finding of Experiment 2, revealed by the error analysis, was that the rats seemed to have had strong biases to turn left or right. Although the analysis of errors was confined, for obvious reasons, to the most unsuccessful rats, it may be inferred that many, if not all, of the rats had such biases. As was discussed earlier, although such biases could help or hinder in the case of response learners, depending on which response was required, and there is some evidence that they helped more than they hindered, all such response biases would have led to $50 \%$ reinforcement and, hence, slower acquisition for all place learners. It is interesting to note that Tolman et al. (1946) required all their response rats to turn right. Of course, it is risky to make inferences about the response tendencies of the rats used by Tolman et al., but on the basis of our data that revealed marked left-turn tendencies, they may have been asking the majority of their response rats to make a response that was contrary to their preference. If so, that would help explain why their response learners did so poorly. It is also interesting to note that Blodgett et al. (1949) required their response rats to turn in a direction opposite to that chosen on their first trial. However, any claim that Blodgett et al. thus put all their response learners at a disadvantage might be countered with the argument that a single instance of choice in a T-maze is insufficient to show clear evidence of a turn preference. On the contrary, if their rats had possessed a real predisposition to go left, as most of ours apparently did, the fact that 5 of 8 were required to go left may have actually put the response learners at a slight advantage.
In the final analysis, it may be the response tendencies possessed by our poorly performing rats that did the most damage to the hypothesis that place learning should be superior to response learning. Even under relatively ideal conditions, our place learners outperformed the response learners only at a marginally significant level. In this regard, it is worth noting that it was Tolman (1932) who first made clear the distinction between learning and performance, arguing that what organisms know and what they $d o$ are not always the same.

The present research was stimulated by an old problem that is tied to some degree to historical debates, and in the ensuing years, there has been much work done in the field of spatial leaning in animals. Cognitive maps continue to be topical, and there is copious evidence that the hippocampus may be the site of such geocentric "pictures" of the environment (O'Keefe \& Nadel, 1978). For example, there is evidence that single hippocampal place cells will fire only when a rat is in a particular spot within a familiar environment (O'Keefe \& Dostrovsky, 1971). Gallistel (1990) has provided a mathematical model designed to show how egocentric knowledge of an environment can be converted into geocentric knowledge of that same environment. He argued that such a cognitive map provides animals such as the rat with detailed knowledge concerning the relative placement of such objects as goals within the larger environment. As a result, once the animal knows where it is in a familiar environment, it knows where everything else is and thus can go to defined places on the basis of this knowledge and does not have to rely entirely on featural cues.

Hull (1943) argued that animals learn particular responses, rather than the generalized expectancies Tolman (1948) called cognitive maps. The movement-produced kinesthesis that was so important to Hull's theory and that, presumably, underlies response learning is currently viewed as one mechanism underlying path integration (Etienne \& Jeffery, 2004). According to Etienne and Jeffery, path integration involves knowledge of where one is in space based on continuously updated information provided by the kinesthetic stimulation generated by movement or by the vestibular stimulation that is generated by rotation of an animal in space. The demonstrated ability of many species, including rodents, to return to their nests after foraging excursions either in the absence of visual cues or in cases in which visual cues have been rearranged suggests a role for such integration in animal navigation (e.g., Etienne, Lambert, Reverdin, \& Teroni, 1993). But Etienne, Berlie, Georgakopoulos, and Mauer (1998) argued that unlike insects, which rely primarily on such internal kinesthetic cues to find their starting point again after a foraging bout, rodents pay more attention to external cues, such as landmarks, in such navigation tasks in order to correct the error that inevitably creeps into the path integration that is based on internal information. Of course, normally, the information that is provided by external, especially visual, cues and the information that is provided by internal kinesthetic feedback are congruent, but Maaswinkel and Whishaw (1999) have shown that when such cues are placed in conflict, vision is trusted in preference to kinesthesis. 
Because rats are capable of using the spatial information provided by cognitive maps or the path integration information supplied through kinesthetic feedback, it is perhaps not surprising that it has been so difficult to determine clearly whether place learning or response learning is easier for rats. As Gallistel (1990) pointed out, the answer can depend on small details of setting or procedure. The issue of how place is defined may be especially crucial. In situations in which place is unambiguousthat is, the same in the apparatus as in the larger environment, as was the case for both Tolman et al. (1946) and the present research - place learners perform much better, albeit not necessarily better, than response learners. However, in situations in which place is static when defined in terms of the larger environment but moves about when it is defined with respect to the more immediate apparatus, place does not provide reliable information, and the rats may be forced to rely more on internal cues, such as those provided by kinesthesis. Although knowledge of where one is, based on movement-generated information, may be less reliable than the geocentric knowledge provided by a cognitive map associated with a stable environment, when one is in an unstable environment that provides unreliable information concerning the location of goals, movement-generated data may be more trustworthy. In such cases, it would not be surprising if animals tended to show more reliance on the feedback provided by their own movements than on their incomplete cognitive map and, hence, demonstrate response learning that is superior to place learning.

In Experiment 2 of the present research, the failure of the group effect to reach statistical significance may be due, at least in part, to the paucity of cues provided by the environment. Both Cheng (1986) and Gallistel (1990) have argued that information related to the geometric shape of environments may be learned in a dedicated system that they have termed the geometric module. Cheng and Cheng and Spetch (1998) have shown that in a rectangular environment in which food is always in one corner, rats seem to encode the basic geometric shape of the environment independently of, and in preference to, distinctive visual featural cues associated with the correct corner. The immediate environment in Experiment 2 of the present research was a circular enclosure. Given a geometric module, the regularity of such an environment would have provided little information concerning the location of the baited tower. If the enclosure had had a less regular shape - for example, a rectangle - it might have favored place learning even more. With respect to the extramaze environment, the footprint of the testing room itself was relatively square, and aside from doors and the sink along the west wall, as well as the freezer, table-mounted cube refrigerator, and white lab coat in the northwest, southeast, and southwest corners, respectively, the extramaze cues were fairly sparse. It is possible that had the testing room also been more rectangular, the place rats might have been assisted by the basic geometry of the setting and, hence, have performed better. Similarly, had the walls in the laboratory been decorated more, the place rats might have been assisted in their efforts to locate the correct tower within the larger space. Of course, just as a minimally discriminable research space might have made the task more difficult for the place rats, it might have actually facilitated learning for the response rats, which might be expected to perform best when not distracted by extramaze cues of any sort.

Considered together, the results of this research suggest that when the meaning of place is not ambiguous, and when the discriminability of starting points is controlled, rats find it at least as easy to go to a particular unchanging spot from different starting points as to make a particular egocentric response that ends in different places regardless of starting point. The evidence also suggests, however, that rats bring strong response biases with them, biases that inevitably interfere with good place learning but that make response learning especially easy in cases in which the required response is the same as the predisposed response and especially difficult in cases in which the required response is not the predisposed response. Finally, the research once again highlights the point that the answer to the question of whether response or place learning is better will always depend on a variety of factors and that even small differences in experimental conditions are likely to affect the outcome.

\section{AUTHOR NOTE}

This research was supported by grants from the Faculty of Arts and Social Sciences at Huron University College. Portions of the present research were presented at various conferences, including the meetings of the Canadian Society for Brain, Behaviour and Cognitive Sciences in St. John's, June 2004, and the joint meeting of the Canadian Society for Brain, Behaviour and Cognitive Sciences and the Experimental Psychology Society of Great Britain in Montreal, July 2005. A.C. is now at the University of Guelph. Correspondence concerning this article should be directed to M. R. Cole, Department of Psychology, Huron University College, University of Western Ontario,1349 Western Road, London, ON, N6G 1H3 Canada (e-mail: mcole@uwo.ca).

\section{REFERENCES}

Blodgett, H. C., McCutchan, K., \& Mathews, R. (1949). Spatial learning in the T-maze: The influence of direction, turn, and food location. Journal of Experimental Psychology, 39, 800-809.

Cheng, K. (1986). A purely geometric module in the rat's spatial representation. Cognition, 23, 149-178.

Cheng, K., \& Spetch, M. (1998). Mechanisms of landmark use in mammals and birds. In S. Healy (Ed.), Spatial representation in animals (pp. 1-17). Oxford: Oxford University Press.

Etienne, A. S., Berlie, J., Georgakopoulos, J., \& Mauer, R. (1998). Role of dead reckoning in navigation. In S. Healy (Ed.), Spatial representation in animals (pp. 54-68). Oxford: Oxford University Press.

ETIENNe, A. S., \& JefFerY, K. J. (2004). Path integration in mammals. Hippocampus, 14, 180-192.

Etienne, A. S., Lambert, S. J., Reverdin, B., \& Teroni, E. (1993). Learning to recalibrate the role of dead reckoning and visual cues in spatial navigation. Animal Learning \& Behavior, 21, 266-280.

Gallistel, C. R. (1990). The organization of learning. Cambridge, MA: MIT Press.

Hull, C. (1943). Principles of behavior. New York: Appleton-CenturyCrofts.

MaAswinkel, H., \& Whishaw, I. (1999). Homing with locale, taxon, and dead reckoning strategies by foraging rats: Sensory hierarchy in spatial navigation. Behavioural Brain Research, 99, 143-152.

Markus, E. J., Qin, Y., Leonard, B., Skaggs, W. E., McNaughton, B. L., \& BARNES, C. A. (1995). Interactions between location and task affect the spatial and directional firing of hippocampal neurons. Journal of Neuroscience, 15, 7079-7094. 
McNaughton, B. L., Barnes, C. A., \& O'Keefe, J. (1983). The contributions of position, direction, and velocity to single unit activity in the hippocampus of freely-moving rats. Experimental Brain Research, 52, 41-49.

Muller, R. U., Kubie, J. L., \& RancK, J. B., JR. (1987). Spatial firing patterns of hippocampal complex-spike cells in a fixed environment. Journal of Neuroscience, 7, 1935-1950.

O'Keefe, J., \& Dostrovsky, J. (1971). The hippocampus as a spatial map: Preliminary evidence from unit activity in the freely-moving rat. Brain Research, 34, 171-175.

O'Keefe, J., \& NAdel, L. (1978). The hippocampus as a cognitive map. Oxford: Oxford University Press, Clarendon Press.

Save, E., Poucet, B., \& Thinus-Blanc, C. (1998). Landmark use and the cognitive map in the rat. In S. Healy (Ed.), Spatial representation in animals (pp. 119-132). Oxford: Oxford University Press.

Skinner, D. M., Etchegary, C. M., Ekert-Maret, E. C., Baker, C. J.,
Harley, C. W., Evans, J. H., \& Martin, G. M. (2003). An analysis of response, direction, and place learning in an open field and T maze. Journal of Experimental Psychology: Animal Behavior Processes, 29, 3-13.

Tolman, E. C. (1932). Purposive behavior in animals and men. New York: Century Company.

Tolman. E. C. (1948). Cognitive maps in rats and men. Psychological Review, 55, 189-208.

Tolman, E. C., Ritchie, B. F., \& Kalish, D. (1946). Studies in spatial learning: II. Place learning versus response learning. Journal of Experimental Psychology, 36, 221-229.

Wike, E. L., Kintsch, W., \& Remple, R. (1959). Selective learning and habit reversal as a function of partial reinforcement during training and reversal. Psychological Reports, 5, 665-668.

(Manuscript received January 26, 2007; revision accepted for publication July 4, 2007.) 